\title{
EXTENDING TRIANGULATIONS
}

M. A. ARMSTRONG

Let $Q$ be a compact piecewise linear (PL) manifold, and $M$ a proper compact PL-submanifold of $Q$. To say $M$ is a proper submanifold means that the boundary $\dot{M}=M \cap \dot{Q}$. A triangulation of $M$ will mean a combinatorial manifold $K$ together with a homeomorphism $k: K \rightarrow M$ that is compatible with the PL-structure of $M$; where no confusion can arise $k$ is usually omitted.

Definition. A triangulation $J$ of $Q$ is said to extend $K$ if, in the diagram

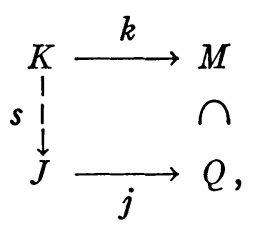

the induced map $s: K \rightarrow J$ is simplicial.

Recall that the submanifold $M$ is said to be locally unknotted in $Q$ if, for some triangulation $K$ of $M$ and extension $J$ of $K$ over $Q$, the closed star ball pair

$$
(\overline{\operatorname{star}}(s v, J), s[\overline{\operatorname{star}}(v, K)])
$$

is unknotted for each vertex $v \in K$. The choice of $K$ and $J$ is irrelevant, since if this is true for a particular pair $K, J$, it is true for any subdivisions $K^{\prime}, J^{\prime}$ and consequently for any other choice. Of course, by [1], local knotting can only occur in codimension 2 , and possibly in codimension 1, depending on the validity or otherwise of the PL Schönflies conjecture.

Theorem. Every triangulation of $M$ can be extended over $Q$ if and only if $M$ is locally unknotted in $Q$.

Corollary 1. Any triangulation of the boundary of a compact PLmanifold can be extended to a triangulation of the whole manifold.

COROLlaRY 2. If $M$, instead of being proper in $Q$, is contained in the interior of $Q$, and if the codimension is $\geqq 3$, then any triangulation of $M$ can be extended over $Q$.

As an example of a nonextendable triangulation in codimension 2, consider the cone on a knotted PL-sphere pair $\left(S^{n+1}, S^{n-1}\right)$. This is a

Received by the editors July 18, 1966. 
ball pair $\left(B^{n+2}, B^{n}\right)$ in which $B^{n}$ is locally knotted at the cone point. Triangulate $B^{n}$ as an $n$-simplex, and suppose this triangulation can be extended to $B^{n+2}$. Then the ball pair consisting of the closed star of $B^{n}$ in this extension, and $B^{n}$ itself, is unknotted, contradicting the local knottedness of the pair $\left(B^{n+2}, B^{n}\right)$.

Proofs of the theorem and its corollaries will follow a couple of elementary lemmas.

Lemma 1. Any triangulation of $M$ has a derived that can be extended over $Q$.

Proof. Let $K$ be a triangulation of $M$. Since the embedding of $M$ in $Q$ is PL, some subdivision $K^{\prime}$ of $K$ can be extended to a triangulation $J$ of $Q$. By [2, Lemma 4], there is a derived $K^{(r)}$ of $K$ that is isomorphic to some subdivision $K^{\prime \prime}$ of $K^{\prime}$. Finally, by the Corollary to Lemma 3 of [2], there is a subdivision $J^{\prime}$ of $J$ such that $j: J^{\prime} \rightarrow Q$ extends $k: K^{(r)} \rightarrow M$.

Lemma 2. Let $(X, Y)$ and $\left(X_{1}, Y_{1}\right)$ be two unknotted $P L$-ball pairs. Then any PL-homeomorphism $h: \dot{X}_{1} \cup Y_{1}, Y_{1} \rightarrow \dot{X} \cup Y, Y$ can be extended to a PL-homeomorphism $\bar{h}: X_{1}, Y_{1} \rightarrow X, Y$.

This result occurs as Lemma 18 in [2].

Proof of the Theorem. Suppose firstly that $M$ is locally unknotted in $Q$. Given a triangulation $K$ of $M$ there is, by Lemma 1, a derived that can be extended over $Q$. Now any derived of a finite complex is obtained by a finite number of stellar subdivisions-each such being the result of starring some simplex at an interior point. Therefore (by induction on the number of stellar subdivisions) it is sufficient to prove that if $\sigma K$ is obtained from $K$ by a single stellar subdivision, and if $\sigma K$ can be extended over $Q$, then $K$ can be extended over $Q$.

Let $\sigma K$ be obtained by starring the simplex $A \in K$ at the interior point $\hat{A}$, and let $J$ be an extension of $\sigma K$ over $Q$, i.e. in

$$
\sigma K \stackrel{k}{\rightarrow} M \subset Q \stackrel{j}{\leftarrow} J
$$

the induced map $s: \sigma K \rightarrow J$ is simplicial. Some further notation is needed; let $L$ be the subcomplex $s(\sigma K)$ of $J, u$ the vertex $s \hat{A}$, and

$$
X=\overline{\operatorname{star}}(u, J), \quad Y=\overline{\operatorname{star}}(u, L) .
$$

Then, since $M$ is a proper locally unknotted submanifold of $Q$, the pair $(X, Y)$ is an unknotted ball pair. It is a cone pair with vertex $u$, and the idea behind the remainder of this proof is simply to replace this pair by a suitable new cone pair-this replacement will have the 
effect of straightening out

$$
s[\overline{\operatorname{star}}(A, K)]
$$

so that $s$ looks linear.

Choose a vertex $v$ of $s \dot{A}$. Without loss of generality the complex $J$ can be assumed to have the following two properties:

(a) $J$ is contained in a Euclidean space $E^{n}$ in such a way that all vertices except $v$ lie in a linear subspace $E^{n-1}$ and $v$ lies in $E^{n}-E^{n-1}$.

$$
\overline{\operatorname{star}}(v, J) \cap X=\overline{\operatorname{star}}(v, X) .
$$

( $J$ is contained in some Euclidean space. Therefore, to accomplish (a), make a small linear move of $v$ into an extra dimension keeping all other vertices of $J$ fixed. If (b) is not satisfied, the remedy is to work throughout with a first derived of $J \bmod L$. The latter is equally well an extension of $\sigma K$ over $Q$, and satisfies (b).) Construct a new complex as follows:

$$
J_{1}=(J-X) \cup(v *[\dot{X}-\operatorname{star}(v, X)]),
$$

where $*$ denotes linear join, and $\operatorname{star}(v, \dot{X})$ the open star of $v$ in $\dot{X}$. Notice the join is well defined by (a), and $J_{1}$ a simplicial complex by (b). It remains to produce a suitable homeomorphism $j_{1}: J_{1} \rightarrow Q$.

To arrange consistency of notation with Lemma 2, let

$$
X_{1}=v *[X-\operatorname{star}(v, X)], \quad Y_{1}=v *[Y-\operatorname{star}(v, Y)] .
$$

Again using local unknottedness, $(\dot{X}, \dot{Y})$ is an unknotted, and therefore locally unknotted, sphere pair. Thus

$$
(\overline{\operatorname{star}}(v, X), \overline{\operatorname{star}}(v, Y))
$$

is an unknotted ball pair, and so the complementary pair in $(\dot{X}, \dot{Y})$ is also unknotted. Consequently $\left(X_{1}, Y_{1}\right)$ is exhibited as the cone on an unknotted ball pair, and is therefore itself unknotted. Let

$$
f: Y_{1} \rightarrow \overline{\operatorname{star}}(A, K)
$$

be the isomorphism defined as the linear extension of $s^{-1}$ on the vertices. Then the identity and the composition

$$
Y_{1} \stackrel{f}{\rightarrow} \overline{\operatorname{star}}(A, K) \stackrel{s}{\rightarrow} Y
$$

together define a PL-homeomorphism $h: \dot{X}_{1} \cup Y_{1}, Y_{1} \rightarrow \dot{X} \cup Y, Y$. By Lemma 2, this may be extended to $\bar{h}: X_{1}, Y_{1} \rightarrow X, Y$. Finally, define $j_{1}: J_{1} \rightarrow Q$ by

$$
j_{1}\left|J_{1}-X_{1}=j, \quad j_{1}\right| X_{1}=j \bar{h} .
$$


Then by construction $j_{1}: J_{1} \rightarrow Q$ extends $k: K \rightarrow M$.

Conversely, suppose $M$ is locally knotted in $Q$. To complete the proof one needs a triangulation of $M$ that cannot be extended over $Q$. Let $x$ be a point at which $M$ is locally knotted in $Q$. Then it is enough to produce a triangulation of $M$ in which

(i) if $x \in M$ (the interior of $M$ ) then $x$ lies in the interior of an $m$ simplex, or

(ii) if $x \in M$, then $x$ lies in the interior of an $(m-1)$-simplex. For let $K$ be such a triangulation, and assume $K$ can be extended to a triangulation $J$ of $Q$. Let $A$ be the simplex of $K$ that has $x$ in its interior, and let $K^{\prime}, J^{\prime}$ result from $K, J$ by starring $A$ at $x$. Then

$$
\left(\operatorname{link}\left(x, J^{\prime}\right), \operatorname{link}\left(x, K^{\prime}\right)\right)=A *(\operatorname{link}(A, J), \operatorname{link}(A, K))
$$

and therefore is an unknotted sphere (ball) pair for $x \in \stackrel{\circ}{M}(\dot{M})$, contradicting the local knotting of $M$ in $Q$ at $x$. Triangulations of the required type can be constructed as follows. Let $k: K \rightarrow M$ be any triangulation of $M$. Choose a point $y$ of $M$ such that $k^{-1} y$ lies in the interior of an $m$-simplex if $x \in \dot{M}$, and in the interior of an $(m-1)$ simplex of $\dot{K}$ if $x \in \dot{M}$. Using the homogeneity of $M$ there is a PLhomeomorphism $h: M \rightarrow M$ that sends $y$ to $x$. Then the triangulation $h k: K \rightarrow M$ has the required property.

The proof of the theorem is now complete.

Proof of Corollary 1 . Let $M$ be the manifold in question. Add a collar to $M$ and denote the resulting manifold by $Q$. Then $\dot{M}$ is a proper locally unknotted submanifold of $Q$ and so the theorem is applicable. Therefore any triangulation of $\dot{M}$ can be extended to a triangulation of $Q$, and of course $M$ must appear as a subcomplex.

Proof of Corollary 2. Suppose $K$ is a given triangulation of $M$, and let $N$ be a relative regular neighbourhood of $M \bmod \dot{M}$ in $Q$. By [1] $M$ is locally unknotted in $N$. First apply the theorem to extend $K$ over $N$, then apply Corollary 1 to the manifold $Q-N$ to complete the extension.

Two questions have been neglected. If $M$ is locally knotted in $Q$, which triangulations of $M$ are extendable over $Q$ ? When is it possible to extend triangulations for polyhedra? Information on both of these will be given in a subsequent paper by E. C. Zeeman [3].

I would like to thank Professor Zeeman for his encouragement.

\section{REFERENCES}

1. E. C. Zeeman, Unknotting combinatorial balls, Ann. of Math. 78 (1963), 501-526.

2. - Seminar on combinatorial topology (mimeographed), Inst. Hautes Etudes Sci., Paris, 1963.

The University of Warwick, Coventry 